\title{
Review of wireless sensors networks in health applications
}

\author{
Enrique Dorronzoro Zubiete \\ Universidad de Sevilla \\ Seville, Spain \\ Email: enriquedz@dte.us.es
}

\author{
Luis Fernandez Luque \\ Northern Research Institute \\ Troms $\emptyset$, Norway \\ Email: luis.luque@norut.no
}

\author{
Ana Verónica Medina Rodríguez \\ and Isabel Gómez González \\ Universidad de Sevilla \\ Seville, Spain
}

\begin{abstract}
Wireless Sensor Networks (WSN) are becoming increasingly important for telemedicine applications, monitoring patients both in the clinical setting and at home. They reduce user discomfort, enhance mobility and reduce costs. WSN are also fundamental in Ambient Assisted Living (AAL) since these smart systems, which are tailored to users needs, collect information about users and their ambient in order to provide personalized feedback.

Despite the growing use of wireless communications in the health domain and in AAL systems there is a lack of research literature reviewing trials of these technologies. This paper provides a systematic review of the use of WSN in the health domain, presenting current WSN implementations. It covers 126 papers, of which 26 are studies, classified according to inclusion criteria. There is presented a discussion about the recent research conducted in the field.
\end{abstract}

\section{INTRODUCTION}

Wireless Sensor Network (WSN) are becoming increasingly important for monitoring patients both in the clinical setting and at home. They provide more comfort for patients, with the absence of wires reducing costs and providing more flexibility. WSN are also fundamental in AAL since these smart systems, tailored to users needs, collect information about users and their ambient in order to provide personalized feedback. Wireless Sensor Networks can integrate vital sign sensors and also environmental sensors such as air quality.

Despite the growing use of wireless communications in the health domain and AAL systems the research literature reviewing trials of these technologies is still scarce. This paper provides a systematic review of the use of WSN in the health domain.

A WSN consists of spatially distributed, autonomous sensors (sensing nodes) that cooperate to monitor physical or environmental conditions, such as temperature, sound, vibration, pressure, etc.

The main components of a WSN are:

- Sensing node: every sensing node is equipped with a microcontroller and a power source. The sensing nodes monitor conditions, conducting small processing operations and transmitting this data to other sensing nodes in the network.

- Transmission technology: to transport data among network nodes. WiFi, Bluetooth, Zig-Bee, and RFID are the most popular transmission technologies. Choosing between them will depend on lifetime maximization, robustness and fault tolerance.
- Standards: to define how data provided by sensing nodes is structured. The use of a data standard benefits system integration and interoperability of the system.

The main reason for using WSN is to improve the quality of healthcare systems. They have several advantages over traditional wired systems[29]: ease of use, reduced risk of infection, reduced risk of failure, reduced user discomfort, enhanced mobility and lower cost of care delivery.

Furthermore, WSN introduce new issues such as lower bandwidth availability, interference, battery life, etc. that have to be considered.

This paper reviews current WSN implementations in the health domain. It provides a systematic review of the use of WSN in health applications, presenting current WSN research implementations. It compares issues and arguments surrounding the different approaches and describes recent research in the field.

Section I describes the methodology used to select and classify papers. Section II presents the main ideas which are then discussed in Section III. Finally, our conclusions are presented.

\section{METHODOLOGY}

This section describes the methodology used to identify and classify the research papers.

\section{A. Establish exclusion criteria}

The most important aspects are to do with results with users. That is why exclusion criteria have been applied to those papers that present a system implementation where a WSN is used.

\section{B. Choosing the search engine}

We used Pubmed, a search engine for health-related publications, which provides results according to the case of study. However, other search engines were used but then ruled out due to the poor results obtained.

For example, using the keywords wireless, sensor health on IEEE xplore returns 50 results, all of which match the exclusion criteria. Using the same keywords at Pubmed returns 126 results, with $21 \%$ of them matching the inclusion criteria.

Search results using different keywords are presented at Table I

- Keywords: parameter used in the search engine. 
TABLE I

SEARCH RESULTS

\begin{tabular}{lrr} 
Keywords & Total & Criteria \\
\hline \hline Wireless, sensor, implementation & 39 & 4 \\
Wireless, sensor, health & 126 & 26 \\
Wireless, sensor, clinical & 53 & 10 \\
\hline \hline total & 218 & 26
\end{tabular}

- Total: number of papers provided from the selected keywords search.

- Criteria: number of papers that match the inclusion criteria.

\section{Establish classification parameters}

The papers that matched the inclusion criteria were classified according to the following parameters:

- Health focus: type of application implemented and presented in the paper.

- Sensors: enumeration of the sensors integrated at the system.

- Transmission technology: used by the sensors to transmit data.

- Autonomy: Battery life.

- N. of participants: number of participants and their health (health or unhealthy).

Table II. shows the classification of each paper according to these parameters.

\section{RESULTS}

This section is divided into four subsections.

\section{A. General implementation strategies}

The general structure implemented in most of the publications is presented in Fig. 1, illustrating two different scenarios:

- Sensors are wired to a personal device which sends the raw or processed data to a personal computer (point to point).

- Sensors are connected using wireless transmission technology to a personal computer that processes the acquired data (point to multipoint).

The general structure implemented in most of the publications is presented at fig. 1 which defines two different scenarios:

- Sensors are connected to a personal device using wires. It is the personal device who sends the acquired data, processed or not, to a personal computer(point to point).

- Sensors are connected using a wireless transmission technology to a personal computer that processes the acquired data(point to multipoint).

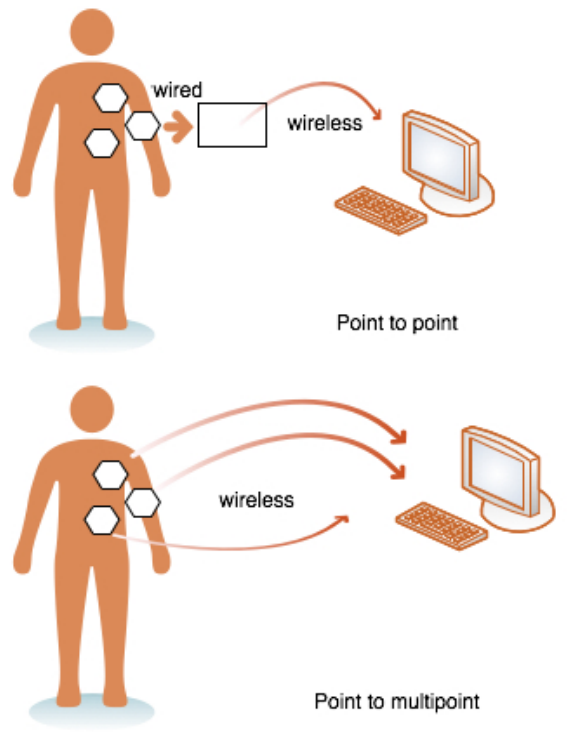

Fig. 1. General system structure

\section{B. Applications}

The reviewed publications can be divided into two main groups of application: monitoring and fall detection.

Fall detection applications cater for a specific scenario where the data is provided by an accelerometer and additional information (i.e. GPS data). Each paper contributes with its own algorithm to determine whether the participant has fallen over. They share a common structure in which the sensor gathers the data from the accelerometer; this information is then processed at the sensor and the fall event is transmitted to a central device where the information is displayed.

Monitoring systems can be divided into those that implement a general health monitoring system and those that provide a solution to a specific condition (diabetes, sleep disorders, etc.). General health monitoring systems focus on system structure and how information is gathered and transmitted, to a central device and displayed. The specific applications present a study about how the information is gathered, mainly using trials with a large number of participants, and they also discuss the results of these trials.

\section{Network and standards}

Two main transmission technologies are used to communicate sensors with WSN, ZigBee and Bluetooth. The main feature of these standards is the low power consumption which is a basic feature of WSN.

There are two main transmission technologies that are used in order to communicate sensors at the WSN, ZigBee and Bluetooth. The main feature about these standards is the low power consumption which is a basic feature at the WSN.

Other alternatives use Radio Frequency (RF)[7], Radio Frequency IDentification (RFID)[23] or they do not even mention the transmission technology[24][27] . 
TABLE II

WSN CLASIFFICATION

\begin{tabular}{|c|c|c|c|c|c|}
\hline Reference & Health focus & Sensors & Trans. technology & Autonomy & N. of participants \\
\hline Biao 2009[2] & Monitoring intestinal motility & Pressure, thermometer, $\mathrm{pH}$ & Propietary & $>180$ hours & 10 (healthy) \\
\hline Campo 2008[3] & Fall detection & Accelerometer, GPS & ZigBee & 15 days & $\mathrm{NA}$ \\
\hline Chung 2008[4] & Monitoring & Accelerometer, ECG, SpO2 & ZigBee & N/A & N/A \\
\hline Dinh 2008[5] & Fall detection & Accelerometer & ZigBee & N/A & 1 (healthy) \\
\hline Figueiredo 2010[7] & Monitoring & ECG, local temperature & Propietary & 90 hours & 1 \\
\hline Greene $2010[8]$ & Gait monitoring & Accelerometer, force-plate & Bluetooth & N/A & 30 (healthy) \\
\hline Istepanian:2009[9] & Diabetes monitoring & Glucose & Bluetooth & N/A & 137 (unhealthy) \\
\hline Kayyali 2008[10] & Sleep disorders monitoring & Polysomnography & Bluetooth & N/A & 10(unhealthy) \\
\hline Kim 2007[11] & Respiratory rate monitoring & Ballistocardiogram & ZigBee & N/A & 4(healthy) \\
\hline Kim 2009[12] & Monitoring & ECG, EOG, EEG & Bluetooth & N/A & 3 (Healthy) \\
\hline Lai 2008[13] & Fall detection & Accelerometer & ZigBee & N/A & 1 \\
\hline Lee $2007[17]$ & Fall detection & Accelerometer & ZigBee & N/A & 30 (Healthy) \\
\hline Lee $2008[18]$ & Fall detection & Accelerometer & Zigbee & N/A & 30 \\
\hline Lee $2009[15]$ & Monitoring & ECG & ZigBee & 24 hours & 1 \\
\hline Lee $2010[14]$ & Monitoring & PPG, accelerometer & ZigBee & N/A & 2(healthy) \\
\hline Lee $2011[16]$ & Fall detection & Accelerometer, ECG & ZigBee & 10 hours & 1 \\
\hline Lou 2010[20] & Scoliosis monitoring & Force transducer & ZigBee & 8 months & 6 (unhealthy) \\
\hline Lou 2010[19] & Scoliosis monitoring & Force transducer & ZigBee & 130 days & 10(unhealthy) \\
\hline Mansouri 2011[21] & Glaucoma monitoring & Intraocular pressure & Bluetooth & N/A & 15 (unhealthy) \\
\hline Morris 2008[22] & Body fluids analysis & $\mathrm{pH}$, Sodium and conductivity & ZigBee & N/A & N/A \\
\hline NG 2010[23] & Temperature monitoring & Thermometer & RFID & 12 months & 109 (unhealthy) \\
\hline Ohki 2007[24] & Endovascular aneurysm repair & Pressure & N/A & RF powered & 76 (healthy) \\
\hline Poh 2010[25] & Monitoring photoplethysmography & Accelerometer & Bluetooth & N/A & 14 \\
\hline Srinivasan $2007[26]$ & Fall detection & Accelerometer & IEEE 802.15 .4 & N/A & 15(healthy) \\
\hline Sugano $2010[27]$ & Monitoring Arrhythmia & ECG & N/A & N/A & 67(healthy) \\
\hline Tawa 2009[28] & Breathing training & Optical, accelerometer & Bluetooth & N/A & \\
\hline
\end{tabular}

On the other hand there is an important issue surrounding data transmission as no data standard is used. Some papers describe their own data structure or do not even mention it.

\section{Trials}

As Table II shows, the number of participants in system testing is low. Only four of the 26 papers presented have more than 30 participants. The main objective of this test is to determine the correct behavior system in short trials. There is no discussion about comfort or long-term monitoring.

\section{DISCUSSION}

Several challenges need to be overcome to enrich current implementations in the WSN domain. These challenges take the form of longer trials, comfort studies, the use of standards, security requirements and reliability. They are discussed in the following subsections.

\section{A. Trials}

More participants and longer trials would allow the measurement of other parameters such as comfort and system autonomy. Comfort is one of the advantages provided by WSN as mentioned in the Introduction. The importance of comfort is discussed in Subsection III-C. Increasing the number of participants would also bolster statistical results.

\section{B. Standards}

When dealing with medical data, several standards are used to structure the information that is sent between medical devices. The WSN publications reviewed in this paper do not use a specific standard for sending data or implementing the services provided by a sensor. Most of the publications do not even mention how the data is sent, while only a few of them implement the solution provided by the manufacturer or a newly defined one.

The lack of any standard turns these solutions into isolated systems because there are many interoperability issues that reduce opportunities for integration in other platforms. A recent standard, IEEE 1451[1], has been designed to standardize communication in WSN; it is not restricted to a single transmission technology, being able to operate with the most popular ones (ZigBee, Bluetooth, etc.). Other proposals use the X73, ISO/IEEE11073 standard, also defined at IEEE, and which was originally designed to provide connectivity between medical devices.

\section{Comfort}

WSN increments the comfort for long-term monitoring. A study is presented at [19] about the effectiveness of orthotic treatment for scoliosis and the importance of time and how well the orthosis is worn. The most commonly recommended wear time is 23 hours/day. This study may tell us how important comfort is in telemonitoring systems. But, as previously mentioned, trials are not usually very 
exhaustive regarding comfort conditions, with most being conducted over short periods. Most of the publications fail to mention system autonomy. In the previous example[19] trials were conducted with unhealthy participants for more than 4 months using a sensor with around 130 days of autonomy.

\section{Security requirements}

WSN requires security methods to authenticate and provide privacy. [30] demonstrates that if sensors cannot be successfully authenticated the incorrect information may be stored in the medical record. Authentication methods can be effective in preventing false data injection and Denial of Service (DoS) attacks. Authenticity, integrity, and confidentiality between biosensors must be guaranteed.

An interesting finding in our research was the lack of papers reporting quality of security aspects. This is surprising since the data collected by wireless sensors may be of vital importance or contain very sensitive information.

In our search strategy we aimed at both home systems and clinical systems, but unfortunately we found mainly examples in the clinical setting. We only found examples referring to possibilities of using WSN for AAL but no trials about implementations. However, wireless communications for AAL have been addressed in the research literature [7][6].

None of the reviewed publications made any considerations about security. The implemented systems do not provide any security about data transmission or authentication methods. The topic is not even discussed.

\section{E. Reliability}

Despite wireless transmission technologies providing their own mechanisms for preventing interference from other wireless sources, transmission reliability must be guaranteed. While some publications [3] mentioned improving reliability as a future objective, this reliability referred to the fall detection mechanism. None of the papers discussed the reliability of the communications implemented in the system.

\section{CONCLussions}

This paper has presented current systems implementations in the WSN domain. There are several applications that focus on telemonitoring and fall detection that use wireless technologies like ZigBee or BlueTooth.

As mentioned, these implementations would benefit from more trials, lasting longer and with more participants to provide more detailed information about system autonomy and comfort. Guaranteeing the privacy and authenticity of the transmitted data is also an important objective. To achieve this, security mechanisms have to be implemented.
Wireless transmission technologies can be affected by different kinds of interference. As the data transmitted in telemedicine systems is sensitive, being able to provide reliability is vital.

As mentioned, one of the most important challenges concerns the use of standards. These standards would help by providing a common interface that would make it possible to integrate these otherwise isolated systems. This is an important goal at AAL where there are many different kinds of sensors. Providing them with a standardized interface would make it easier to integrate and coordinate them.

\section{ACKNOWLEDGMENTS}

This study has been carried out at the Northern Research Institute (NORUT) with the support of IS-MOBIL by the Research Council of Norway and within the framework of two research programs: (p08-TIC-3631) Multimodal Wireless Interface (IMI) funded by the Regional Government of Andalusia and Efficient and Health Intelligent Technologies Oriented to Health and Comfort in Interior Environments (TECNO-CAI), a project approved in the fifth edition of the CENIT program run by Spanish Ministry of Science and Innovation.

\section{REFERENCES}

[1] Ieee standard for a smart transducer interface for sensors and actuators - common functions, communication protocols, and transducer electronic data sheet (teds) formats. IEEE Std 1451.0-2007, pages 1 $-335,2007$.

[2] H Biao, Y Guozheng, and Z Peng. Multi-sensor radiotelemetry system for intestinal motility measurement. J Med Eng Technol, 33(1):66-71, Jan 2009.

[3] Eric Campo and Etienne Grangereau. Wireless fall sensor with gps location for monitoring the elderly. Conf Proc IEEE Eng Med Biol Soc, 2008:498-501, Jan 2008.

[4] Wan-Young Chung, Young-Dong Lee, and Sang-Joong Jung. A wireless sensor network compatible wearable u-healthcare monitoring system using integrated ecg, accelerometer and spo2. Conf Proc IEEE Eng Med Biol Soc, 2008:1529-32, Jan 2008.

[5] A Dinh, D Teng, L Chen, S B Ko, Y Shi, J Basran, and V Del BelloHass. Data acquisition system using six degree-of-freedom inertia sensor and zigbee wireless link for fall detection and prevention. Conf Proc IEEE Eng Med Biol Soc, 2008:2353-6, Jan 2008.

[6] Francisco J Fernandez-Luque, Juan Zapata, and Ramon Ruiz. A system for ubiquitous fall monitoring at home via a wireless sensor network. Conf Proc IEEE Eng Med Biol Soc, 1:2246-9, Jan 2010.

[7] C P Figueiredo, K Becher, K P Hoffmann, and P M Mendes. Low power wireless acquisition module for wearable health monitoring systems. Conf Proc IEEE Eng Med Biol Soc, 1:704-7, Jan 2010.

[8] Barry R Greene, Denise McGrath, Karol J O’Donovan, Ross O’Neill, Adrian Burns, and Brian Caulfield. Adaptive estimation of temporal gait parameters using body-worn gyroscopes. Conf Proc IEEE Eng Med Biol Soc, 1:1296-9, Jan 2010.

[9] Robert S H Istepanian, Karima Zitouni, Diane Harry, Niva Moutosammy, Ala Sungoor, Bee Tang, and Kenneth A Earle. Evaluation of a mobile phone telemonitoring system for glycaemic control in patients with diabetes. J Telemed Telecare, 15(3):125-8, Jan 2009. 
[10] Hani A Kayyali, Sarah Weimer, Craig Frederick, Christian Martin, Del Basa, Jesse A Juguilon, and Felicitas Jugilioni. Remotely attended home monitoring of sleep disorders. Telemed J E Health, 14(4):371-4, May 2008.

[11] Jong-Myoung Kim, Joo-Hyun Hong, Myeong-Chan Cho, Eun-Jong Cha, and Tae-Soo Lee. Wireless biomedical signal monitoring device on wheelchair using noncontact electro-mechanical film sensor. Conf Proc IEEE Eng Med Biol Soc, 2007:574-7, Jan 2007.

[12] Youn Sung Kim, Hyun Jae Baek, Jung Soo Kim, Haet Bit Lee, Jong Min Choi, and Kwang Suk Park. Helmet-based physiological signal monitoring system. Eur J Appl Physiol, 105(3):365-72, Feb 2009.

[13] Daniel T H Lai, E Charry, R Begg, and M Palaniswami. A prototype wireless inertial-sensing device for measuring toe clearance. Conf Proc IEEE Eng Med Biol Soc, 2008:4899-902, Jan 2008.

[14] E M Lee, J Y Shin, J H Hong, E J Cha, and T S Lee. Glass-type wireless ppg measuring system. Conf Proc IEEE Eng Med Biol Soc, 1:1433-6, Jan 2010.

[15] Sangjoon Lee, Jinkwon Kim, Jungkuk Kim, and Myoungho Lee. A design of the u-health monitoring system using a nintendo ds game machine. Conf Proc IEEE Eng Med Biol Soc, 2009:1695-8, Jan 2009.

[16] Sangjoon Lee, Jungkuk Kim, and Myoungho Lee. The design of the m-health service application using a nintendo ds game console. Telemed J E Health, Jan 2011.

[17] Youngbum Lee, Jinkwon Kim, Muntak Son, and Myoungho Lee. Implementation of accelerometer sensor module and fall detection monitoring system based on wireless sensor network. Conf Proc IEEE Eng Med Biol Soc, 2007:2315-8, Jan 2007.

[18] Youngbum Lee and Myoungho Lee. Accelerometer sensor module and fall detection monitoring system based on wireless sensor network for e-health applications. Telemed J E Health, 14(6):587-92, Aug 2008.

[19] Edmond Lou, Doug Hill, Douglas Hedden, Jim Mahood, Marc Moreau, and Jim Raso. An objective measurement of brace usage for the treatment of adolescent idiopathic scoliosis. Med Eng Phys, Nov 2010.

[20] Edmond Lou, Doug L Hill, and James V Raso. A wireless sensor network system to determine biomechanics of spinal braces during daily living. Med Biol Eng Comput, 48(3):235-43, Mar 2010.

[21] Kaweh Mansouri and Tarek Shaarawy. Continuous intraocular pressure monitoring with a wireless ocular telemetry sensor: initial clinical experience in patients with open angle glaucoma. The British journal of ophthalmology, Jan 2011.

[22] Deirdre Morris, Benjamin Schazmann, Yangzhe Wu, Shirley Coyle, Sarah Brady, Cormac Fay, Jer Hayes, King Tong Lau, Gordon Wallace, and Dermot Diamond. Wearable technology for bio-chemical analysis of body fluids during exercise. Conf Proc IEEE Eng Med Biol Soc, 2008:5741-4, Jan 2008.

[23] Kim-Gau Ng, Sook-Thow Wong, Soh-Min Lim, and Zenton Goh. Evaluation of the cadi thermosensor wireless skin-contact thermometer against ear and axillary temperatures in children. $J$ Pediatr Nurs, 25(3):176-86, Jun 2010

[24] Takao Ohki, Kenneth Ouriel, Pierre Galvagni Silveira, Barry Katzen, Rodney White, Frank Criado, and Edward Diethrich. Initial results of wireless pressure sensing for endovascular aneurysm repair: the apex trial-acute pressure measurement to confirm aneurysm sac exclusion. J Vasc Surg, 45(2):236-42, Feb 2007.

[25] Ming-Zher Poh, Nicholas C Swenson, and Rosalind W Picard. Motiontolerant magnetic earring sensor and wireless earpiece for wearable photoplethysmography. IEEE Trans Inf Technol Biomed, 14(3):78694, May 2010.

[26] Soundararajan Srinivasan, Jun Han, Dhananjay Lal, and Aca Gacic. Towards automatic detection of falls using wireless sensors. Conf Proc IEEE Eng Med Biol Soc, 2007:1379-82, Jan 2007.

[27] Hiroto Sugano, Tetsuo Tsujioka, Tadayuki Inoue, Shigeyoshi Nakajima, Shinsuke Hara, Hajime Nakamura, and Kazuhide Takeuchi. Clinical tests and evaluations of a wireless ecg sensor for realization of ubiquitous health care systems. Conf Proc IEEE Eng Med Biol Soc, 1:2030-3, Jan 2010.

[28] Hiroki Tawa, Yoshiharu Yonezawa, Hiromichi Maki, Hidekuni Ogawa, Ishio Ninomiya, Kouji Sada, Shingo Hamada, and W Morton Caldwell. A wireless breathing-training support system for kinesitherapy. Conf Proc IEEE Eng Med Biol Soc, 2009:5179-82, Jan 2009.

[29] K Townsend, J Haslett, T Tsang, M El-Gamal, and K Iniewski. Recent advances and future trends in low power wireless systems for medical applications. System-on-Chip for Real-Time Applications, 2005. Proceedings. Fifth International Workshop on, pages $476-481$, 2005.

[30] Lin Yao, Bing Liu, Kai Yao, Guowei Wu, and Jia Wang;. An ecgbased signal key establishment protocol in body area networks. Ubiquitous Intelligence \& Computing and 7th International Conference on Autonomic \& Trusted Computing (UIC/ATC), 2010 7th International Conference on, pages 233 - 238, 2010. 